\title{
Can modern infrared analyzers replace gas chromatography to measure anesthetic vapor concentrations? Jan FA Hendrickx ${ }^{* 1}$, Hendrikus JM Lemmens ${ }^{1}$, Rik Carette ${ }^{2}$, Andre M De
Wolf $^{3}$ and Lawrence J Saidman ${ }^{1}$
}

Address: ${ }^{1}$ Department of Anesthesia, Stanford University School of Medicine, Stanford, California, USA, ${ }^{2}$ Anesthesiologist, Department of Anesthesia, OLV Hospital, Aalst, Belgium and '3Department of Anesthesiology, Feinberg School of Medicine, Northwestern University, Chicago, Illinois, USA

Email: Jan FA Hendrickx* - jcnwahendrickx@yahoo.com; Hendrikus JM Lemmens - hlemmens@stanford.edu; Rik Carette - rikcarette@yahoo.com; Andre M De Wolf - a-dewolf@northwestern.edu; Lawrence J Saidman - lsaidman@stanford.edu

* Corresponding author

Published: 8 February 2008

BMC Anesthesiology 2008, 8:2 doi:10.1 |86//47|-2253-8-2
Received: 10 September 2007

Accepted: 8 February 2008

This article is available from: http://www.biomedcentral.com/I47I-2253/8/2

(c) 2008 Hendrickx et al; licensee BioMed Central Ltd.

This is an Open Access article distributed under the terms of the Creative Commons Attribution License (http://creativecommons.org/licenses/by/2.0), which permits unrestricted use, distribution, and reproduction in any medium, provided the original work is properly cited.

\begin{abstract}
Background: Gas chromatography (GC) has often been considered the most accurate method to measure the concentration of inhaled anesthetic vapors. However, infrared (IR) gas analysis has become the clinically preferred monitoring technique because it provides continuous data, is less expensive and more practical, and is readily available. We examined the accuracy of a modern IR analyzer (M-CAiOV compact gas IR analyzer (General Electric, Helsinki, Finland) by comparing its performance with GC.
\end{abstract}

Methods: To examine linearity, we analyzed 3 different concentrations of 3 different agents in $\mathrm{O}_{2}$ : $0.3,0.7$, and $\mathrm{I} .2 \%$ isoflurane; $0.5, \mathrm{I}$, and $2 \%$ sevoflurane; and I, 3 , and $6 \%$ desflurane. To examine the effect of carrier gas composition, we prepared mixtures of I\% isoflurane, I or $2 \%$ sevoflurane, or $6 \%$ desflurane in $100 \% \mathrm{O}_{2}\left(=\mathrm{O}_{2}\right.$ group); $30 \% \mathrm{O}_{2}+70 \% \mathrm{~N}_{2} \mathrm{O}\left(=\mathrm{N}_{2} \mathrm{O}\right.$ group), $28 \% \mathrm{O}_{2}+66 \% \mathrm{~N}_{2} \mathrm{O}$ $+5 \% \mathrm{CO}_{2}\left(=\mathrm{CO}_{2}\right.$ group), or air. To examine consistency between analyzers, four different $\mathrm{M}$ $\mathrm{CAiOV}$ analyzers were tested.

Results: The IR analyzer response in $\mathrm{O}_{2}$ is linear over the concentration range studied: IR isoflurane \% $=-0.0256+(1.006 * G C \%), R=0.998 ;$ IR sevoflurane $\%=-0.008+(0.946 * G C \%)$, $\mathrm{R}=0.993 ;$ and $\mathrm{IR}$ desflurane $\%=0.256+(0.919 * \mathrm{GC} \%), \mathrm{R}=0.998$. The deviation from GC calculated as ( $100 *(\mathrm{IR}-\mathrm{GC}) / \mathrm{GC})$, in \%) ranged from $-\mathrm{II}$ to $11 \%$ for the medium and higher concentrations, and from -20 to $+20 \%$ for the lowest concentrations. No carrier gas effect could be detected. Individual modules differed in their accuracy $(p=0.004)$, with differences between analyzers mounting up to $12 \%$ of the medium and highest concentrations and up to $25 \%$ of the lowest agent concentrations.

Conclusion: M-CAiOV compact gas IR analyzers are well compensated for carrier gas crosssensitivity and are linear over the range of concentrations studied. IR and GC cannot be used interchangeably, because the deviations between GC and IR mount up to $\pm 20 \%$, and because individual analyzers differ unpredictably in their performance. 


\section{Background}

Gas chromatography (GC) has often been considered the most accurate method to measure the concentration of potent inhaled anesthetics (further referred to as "anesthetic vapors"). Infrared (IR) gas analysis provides continuous data and is more readily available clinically, less expensive and more practical, but needs complex calibration and compensation procedures to minimize or eliminate the effects of overlapping IR absorption spectra of anesthetic vapors, $\mathrm{H}_{2} \mathrm{O}, \mathrm{N}_{2} \mathrm{O}$, and $\mathrm{CO}_{2}$ and those of "collision broadening" or "pressure broadening" [1]. The effect of $\mathrm{H}_{2} \mathrm{O}$ has "virtually been eliminated" by the use of Nafion $^{\mathrm{TM}}$ tubing [2,3], except possibly for the older agent halothane [4]. The effect of $\mathrm{N}_{2} \mathrm{O}$ is small, being reported as absent [4], less than 0.01 [5] or -.03 volume vol \% with isoflurane and enflurane [2]. The effect of $\mathrm{CO}_{2}$ is absent [2] or less than $+/-0.01$ vol\% [3,5]. Most authors consider the response of these older IR analyzers to be sufficiently accurate and linear for clinical purposes, but some departure from linearity was found. The Datex Normac "underread" enflurane and isoflurane concentrations in the low range and "over-read" them at the higher range, with the greatest error $6 \%$ of reading [2]. The Datex Capnomac Ultima under-predicted the concentrations of the agents by 10 to $12 \%[3]$.

While the issue of cross-sensitivity of other gases on IR analysis of anesthetic vapors has been resolved, extrapolating the accuracy of these older monitors to modern gas analyzers is complex. First, none of previous studies rigorously examined the performance with sevoflurane or desflurane. Second, in the manufacturing process, each module and each individual filter is compensated for cross gas effects. While this would suggest that analyzers might differ in their performance because the degree of compensation might differ, the issue has not been addressed in older studies because they only examined one analyzer. To assess instrumental variances, data were therefore obtained from $4 \mathrm{M}$-CAiOV compact IR gas analyzers (General Electric [Datex-Ohmeda], Helsinki, Finland)[6]. Finally, modern IR analyzers use a different part of the IR spectrum. The M-CAiOV unit uses the 8 to $9 \mu \mathrm{m}$ range to eliminate the effect of $\mathrm{CO}_{2}$ and to minimize the effect of $\mathrm{N}_{2} \mathrm{O}$. The use of 5 wavelengths between 8 to $9 \mu \mathrm{m}$ allows automated agent detection and a correction factor for the effect of $\mathrm{N}_{2} \mathrm{O}$ for each of these 5 wavelengths. We were particularly interested in the performance of the $\mathrm{M}$ CAiOV unit [6] because its ability to measure vapor concentrations accurately in clinical studies has not been determined [7]. We therefore studied the performance of 4 units, and examined whether IR accuracy approaches that of GC.

\section{Methods}

All gas mixtures were prepared with a conventional anesthesia machine (ADU Delivery Unit by GE, Helsinki, Finland), and sampled from the common gas outlet. For each analyzer, test mixtures were sampled from the common gas outlet at different times; because vaporizer output (and thus the concentration of vapor at the common gas outlet) may differ slightly from moment to moment, test mixtures may differ slightly between analyzers. For the $\mathrm{CO}_{2}$ groups, $5 \mathrm{~mL} \mathrm{CO}$ (from an E-cylinder) was added to a volume of $95 \mathrm{~mL}$ mixture of agent with $30 \% \mathrm{O}_{2}$ $+70 \% \mathrm{~N}_{2} \mathrm{O}$, yielding approximately $5 \% \mathrm{CO}_{2}$ in the final mixture. All samples were drawn into $100 \mathrm{~mL}$ glass syringes. To ensure adequate mixing of the gases, the mixtures were injected via a three-way stopcock at least 4 times into a second $100 \mathrm{~mL}$ glass syringe. Immediately after mixing, the first and last $10 \mathrm{~mL}$ were injected into the gas chromatograph; the mid portion, $80 \mathrm{~mL}$, was sampled by the IR analyzer. When the GC peak height of the first and last $10 \mathrm{~mL}$ sample varied by more than $1 \mathrm{~mm}$, the measurements were repeated. Over the entire course of the study, repeat measures were done on three occasions because operator error had caused gross errors.

A flame ionization detector GC (Gow-Mac 580; Gow-Mac, Bethlehem, PA) was used, equipped with a $4.57 \mathrm{~m}, 22-$ $\mathrm{mm}$ internal diameter column containing $10 \%$ SF-96 on WHP, 68/80 mesh, maintained at $43^{\circ} \mathrm{C}$ with an $8 \mathrm{~mL} /$ min $\mathrm{N}_{2}$ carrier flow. The detector (at $122^{\circ} \mathrm{C}$ ) received $\mathrm{H}_{2}$ at $20 \mathrm{~mL} / \mathrm{min}$ and air at $200 \mathrm{~mL} / \mathrm{min}$. The GC was calibrated before and at intervals during each test using secondary (cylinder) calibration standards. The same calibration gas (one for each agent) was used throughout the study. The calibration gas comes out of a stainless steel cylinder which contains a known concentration of vapor; for each agent, a different cylinder was prepared. The mixture is prepared by creating a subatmospheric pressure in the cylinder. Next, an amount of liquid agent is aspirated into the cylinder. This amount is calculated such that after vaporization the calculated pressure in the cylinder will remain below the vapor pressure of the agent. The cylinder is then filled/pressurized with air. To mix its contents, the cylinder is rolled several times, and subsequently heated overnight by applying an external heat pad. The exact concentration of this secondary standard is determined by comparing it with a primary volumetric standard using gas chromatography. The primary standard is prepared by injecting a known exact amount of liquid agent (measured by weight or glass micropipette) in a glass Erlenmeyer of known volume, taking the effect of temperature into account. Using this primary standard, it was determined that the concentrations of isoflurane, sevoflurane, and desflurane in the tank was 0.744, 1.49, and $4.20 \%$, respectively. Over the entire course of the 
study, the peak height with the same calibration gas for isoflurane $(0.744 \%)$, sevoflurane $(1.49 \%)$, and desflurane $(4.20 \%)$ was $54.0 \mathrm{~mm}$ (standard deviation $1.1 \mathrm{~mm}$ ) for isoflurane, $52.2 \mathrm{~mm}$ (standard deviation $2.0 \mathrm{~mm}$ ) for sevoflurane, and $54.1 \mathrm{~mm}$ (standard deviation $1.4 \mathrm{~mm}$ ) for desflurane; the coefficients of variation for isoflurane, sevoflurane, and desflurane therefore were 2.0, 3.8, and $2.6 \%$ respectively. These calculations are based on 6 calibrations, 3 before and 3 after measuring each agent for each analyzer (total of 18 per analyzer).

The 4 multi-gas analyzers were calibrated using the appropriate calibration gas provided (General Electric [DatexOhmeda], Helsinki, Finland), and as recommended analyses were started no sooner than $30 \mathrm{~min}$ after inserting the module.

\section{Statistical analysis}

Repeated injections of a given sample from a tank or flask containing known volumetric standards into a GC give values with a standard deviation of less than $2-3 \%$ (personal communication with Dr. Eger EI II, and corroborated by our own findings in this study - coefficients of variation for isoflurane, sevoflurane, and desflurane of $2.0,3.8$, and $2.6 \%$ respectively). Because GC is a calibrated reference standard, it can be considered to be an accurate measure of the concentrations within the known limits of accuracy. IR is therefore compared directly to the GC measurements, using linear regression analysis to examine the linearity of the response. To examine the effect of carrier gas and whether analyzers differed in their performance, we used two way ANOVA (factors = carrier gas and analyzer) with the Holm-Sidak method.

\section{Results}

Linear regression results are presented in figure 1 . The following mathematical expression described the response of the IR analyzer, with the standard error of the 2 linear regression parameters between square brackets: IR isoflurane $\%=-0.026+(1.006 *$ GC $\%), \mathrm{R}=0.998,[0.014$; $0.0185] ;$ IR sevoflurane $\%=-0.008+(0.946 * \mathrm{GC} \%), \mathrm{R}=$ 0.993, [0.044; 0.034]; IR desflurane $\%=0.256+(0.919 *$ $\mathrm{GC} \%), \mathrm{R}=0.998,[0.060 ; 0.014]$. The deviation from GC calculated as $\left(100^{*}\right.$ [IR-GC]/GC) for the medium and high concentrations ranged from -9 to $6 \%$ for isoflurane, from -11 to $5 \%$ for sevoflurane, and from -9 to $11 \%$ for desflurane. Deviation was more pronounced with the lower concentrations: from -18 to $2 \%$ for isoflurane, from -20 to $0 \%$ for sevoflurane, and from -8 to $21 \%$ for desflurane.

The difference between GC and IR analysis of volatile anesthetics was not affected by carrier gas $(P=0.942)$, but individual modules differed in their accuracy $(\mathrm{p}=0.004)$, with the differences as great as $25 \%$ with the lowest agent concentrations and as great as $12 \%$ with the middle and highest concentrations (Table 1).

\section{Discussion}

In this study, we confirm that IR analysis of anesthetic vapors by a polychromatic IR analyzer is not affected by carrier gas composition. The response by the IR analyzer is linear for the three agents over the concentration range tested $(\mathrm{R}>0.99)$, but it tends to underestimate the concentrations of isoflurane and sevoflurane and those of desflurane at higher concentrations. The response differs unpredictably between modules.
A

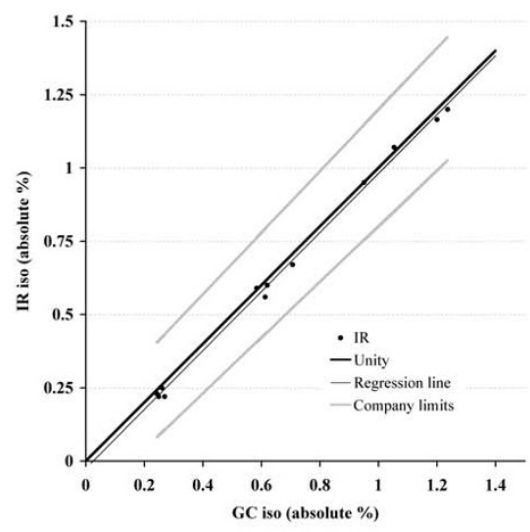

B

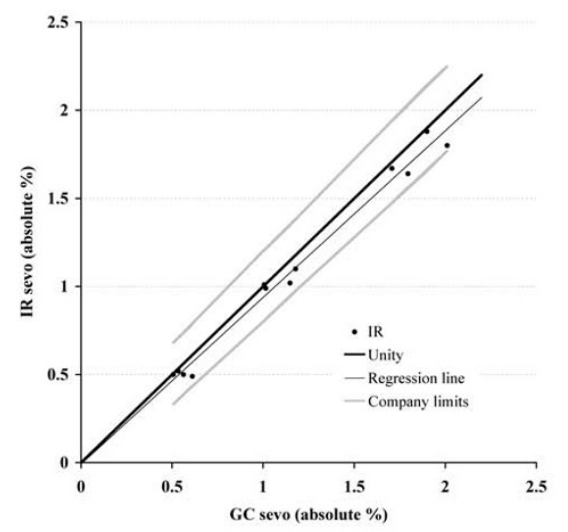

C

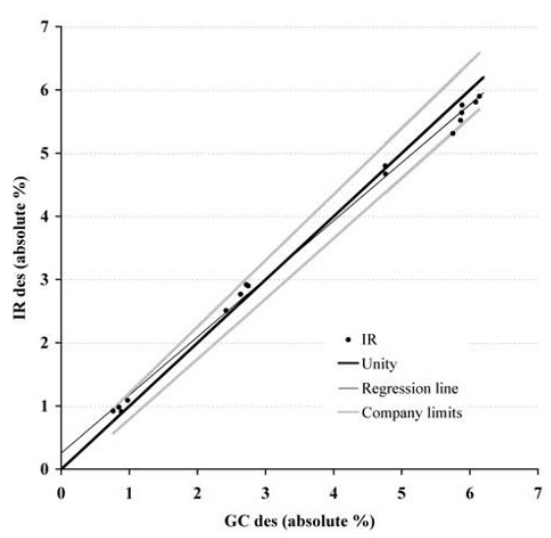

\section{Figure I}

Linear regression plots comparing IR with GC analysis of isoflurane $(A)$, sevoflurane $(B)$, and desflurane $(C)$ in $\mathrm{O}_{2}$ over a range of concentrations. Closed circles = individual data points; thick black line = line of unity; thin grey line = linear regression (see text for formulas), thick grey lines = upper and lower accuracy limits according to company. The performance of the four modules conformed to that specified by the company, $+/-(0.15 \%+5 \%$ of IR reading). 
Table I: Carrier gas effect.

\begin{tabular}{|c|c|c|c|c|c|c|c|c|c|}
\hline \multirow[b]{2}{*}{ Agent } & \multirow[b]{2}{*}{ Analyzer \# } & \multicolumn{2}{|c|}{$\mathrm{O}_{2}$} & \multicolumn{2}{|c|}{$\mathrm{CO}_{2}$} & \multicolumn{2}{|c|}{$\mathbf{N}_{2} \mathrm{O}$} & \multicolumn{2}{|c|}{$\mathrm{N}_{2} \mathrm{O}+\mathrm{CO}_{2}$} \\
\hline & & GC & IR & GC & IR & GC & IR & GC & IR \\
\hline \multirow[t]{4}{*}{ isoflurane } & $\mathbf{I}$ & 1.24 & 1.20 & 1.18 & 1.15 & 1.19 & 1.15 & 1.22 & 1.20 \\
\hline & II & 1.05 & 1.07 & 0.97 & 1.01 & 1.08 & 1.10 & 1.18 & 1.22 \\
\hline & III & 0.95 & 0.95 & 0.88 & 0.86 & 1.24 & 1.30 & 1.11 & 1.12 \\
\hline & IV & 1.20 & 1.17 & 0.85 & 0.81 & 1.14 & 1.06 & 1.08 & 1.00 \\
\hline \multirow[t]{4}{*}{ sevoflurane } & I & 1.90 & 1.88 & 1.96 & 1.90 & 0.91 & 0.93 & 0.88 & 0.88 \\
\hline & II & 2.01 & 1.80 & 1.08 & 0.99 & 1.05 & 0.98 & 0.98 & 0.89 \\
\hline & III & 1.71 & 1.67 & 1.82 & 1.80 & 0.95 & 0.91 & 1.03 & 1.00 \\
\hline & IV & 1.80 & 1.64 & 1.80 & 1.64 & 1.05 & 0.98 & 0.84 & 0.77 \\
\hline \multirow[t]{4}{*}{ desflurane } & $\mathbf{I}$ & 6.14 & 5.90 & 5.00 & 5.00 & 5.95 & 6.03 & 5.71 & 5.40 \\
\hline & II & 5.88 & 5.64 & 5.38 & 5.27 & 5.86 & 5.56 & 5.48 & 5.37 \\
\hline & III & 4.76 & 4.80 & 6.12 & 5.96 & 6.76 & 6.47 & 6.02 & 5.54 \\
\hline & IV & 5.75 & 5.31 & 5.29 & 5.00 & 5.85 & 5.40 & 5.65 & 5.30 \\
\hline
\end{tabular}

Carrier gas composition did not affect IR analysis $(P>0.05)$, but individual modules within the carrier gas group differed in their accuracy $(P=$ 0.004). GC = gas chromatography (measured value, \% of atm); IR = infrared values (measured value, \% of atm).

Inhaled anesthetic vapors absorb IR, allowing their concentration to be measured using the law of Beer-Lambert. More specifically, they absorb IR at $3.3 \mu \mathrm{m}$ and in the 8 to $9 \mu \mathrm{m}$ range. Because of the proximity of a $\mathrm{CO}_{2}$ absorption band at $3.3 \mu \mathrm{m}$ used by older analyzers, $\mathrm{CO}_{2}$ interfered with the measurement of anesthetic vapors. Even though the older analyzers compensated for this effect, the use of five different wavelengths in the 8 to $9 \mu \mathrm{m}$ range eliminates this issue completely.

When $\mathrm{N}_{2} \mathrm{O}$ is present, overlapping absorption spectra complicate the measurement of anesthetics agents. The effect is reduced by the use of the 8-9 $\mu \mathrm{m}$ range, but there still is a need for cross compensation caused by the absorption of $\mathrm{N}_{2} \mathrm{O}$ at the wavelengths used for anesthetic agents. The magnitude of the effect of $\mathrm{N}_{2} \mathrm{O}$ on anesthetic agent measurements depends on the individual characteristics of the optical filters in the IR-sensor of a module. In the manufacturing process, each module and each individual filter is compensated for cross gas effects with $\mathrm{N}_{2} \mathrm{O}$ (personal communication with K. Karlsson, GE Healthcare). Interactions with $\mathrm{N}_{2} \mathrm{O}$ are tested with anesthetic agents, and the modules are then compensated for this effect. The compensation coefficients are stored in the EEPROM-memory of each module. After this compensation, the error caused by $79 \% \mathrm{~N}_{2} \mathrm{O}$ to the zero point of the anesthetic gas measurements must be smaller than +/$0.1 \%$ halothane in order to have the module accepted from this calibration phase. Because the M-CAiOV module measures $\mathrm{N}_{2} \mathrm{O}$ simultaneously with anesthetic agent, it is possible to use a real time compensation for $\mathrm{N}_{2} \mathrm{O}$ in the calculation of the anesthetic agent concentration: instead of assuming a fixed $\mathrm{N}_{2} \mathrm{O}$-concentration, the com- pensation uses the measured real time value of $\mathrm{N}_{2} \mathrm{O}$ as the input value. The complex calibrations and compensations in commercial sensors are proprietary, but they appear to be effective since we could not document an effect of $\mathrm{N}_{2} \mathrm{O}$ on the IR analysis of anesthetic vapors.

The effect of $\mathrm{H}_{2} \mathrm{O}$ vapor is minimized by equalizing the $\mathrm{H}_{2} \mathrm{O}$ vapor pressure in the sample to that in the atmosphere by using Nafion $^{\mathrm{TM}}$ tubing[8]. The technology was already incorporated in older IR analyzers, and found to be effective $[2,3]$. Nafion ${ }^{\mathrm{TM}}$ is a copolymer of tetrafluoroethylene (Teflon ${ }^{\circledast}$ ) and perfluoro-3,6-dioxa-4-methyl-7octene-sulfonic acid). Sulfonic acid (-SO3H) has a high water-of-hydration, absorbing up to 13 molecules of $\mathrm{H}_{2} \mathrm{O}$ for every sulfonic acid group in the polymer. Unlike micro-porous membrane permeation, which transfers water through a relatively slow diffusion process, Nafion $^{\mathrm{TM}}$ removes water by absorption as water-of-hydration, a first order kinetic reaction that equilibrates within milliseconds. $\mathrm{H}_{2} \mathrm{O}$ binds to the sulfonic acid in $\mathrm{Nafion}^{\mathrm{TM}}$ and will readily permeate through the polymer, thus equalizing the humidity of the gas going into the gas sensors with that of the ambient air. This is necessary for two reasons. First, calibration gas is dry, while patient samples have $100 \%$ relative humidity. Calibrating the sensor with dry gas and measuring saturated gas would cause an additional error to the measurements. Secondly, the sample gas coming from the patient circuit needs to pass the Nafion tube so that the humidity equals the ambient humidity before being analyzed. The gas concentrations are thus measured at the prevailing ambient humidity. If the ambient humidity varies very much between the cali- 
bration and the actual monitoring, there will be an additional error component caused by $\mathrm{H}_{2} \mathrm{O}$.

What are the implications of our findings? Effects of crosssensitivity by different gases are virtually absent, a finding we confirmed for $\mathrm{CO}_{2}, \mathrm{~N}_{2} \mathrm{O}, \mathrm{N}_{2}$, and $\mathrm{O}_{2}$. The performance of the four modules conformed to that specified by the company, $+/-(0.15 \%+5 \%$ of IR reading) (figure 1$)$. The measurement accuracy of all tested modules is better than that required by the ISO 21647 standard for essential performance of respiratory gas monitors (ISO21647:2004(E)). Of the 4 analyzers we tested, IR slightly underestimates most anesthetic vapor concentrations, possibly causing the anesthesiologist to administer a slightly greater concentration. This may be more clinically relevant at the lower concentration range. At high concentrations, well above MAC awake and even MAC, the patient is adequately anesthetized and the dose given becomes a titration against vital sign fluctuations. At low concentrations however, in the MACawake range, one is more concerned about the potential for recall, especially in patients who cannot sustain hemodynamic stability in the presence of anesthetic vapors. A measurement that is lower than the actual concentration may lead the clinician to increase the concentration unnecessarily and/or use a vasopressor to maintain adequate blood pressure for the given depth of anesthesia. An error on the high side may lead to inadequate vapor delivery to prevent recall.

Can IR gas analysis be used for clinical research? Even though the use of different IR absorption bands by the MCAiOV compact multi-gas analyzer has allowed automated agent detection and may technically have facilitated the compensation for cross-sensitivity between anesthetic vapors and other gases, it has not improved accuracy of vapor analysis beyond that of existing, older IR analyzers. Deviations from GC calculated as (100* [IRGC]/GC) range from $-11 \%$ to $11 \%$ around 0.5 MAC values, and higher still at lower concentrations. We also found that individual modules differ unpredictably in their accuracy, and that, even though the response is linear, some additional gain (sevoflurane) and offset (isoflurane) control or both (desflurane) may be needed. Finally, small effects of carrier gases can still be present (personal communication with K. Karlsson, GE Healthcare). A recent study by Peyton suggested that the accuracy and precision of measurement of volatile anesthetic gas partial pressures in blood by a double headspace equilibration technique, using a clinical infrared gas analyzer, were comparable to that achieved by previous studies using gas chromatography [9]. However, only one gas analyzer was examined. Because our study demonstrates difference in performance between individual units, our study suggests that GC remains the method of choice to measure absolute concentrations. An alternative interpre- tation of Peyton's findings might be that IR analysis could be used if the performance characteristics of the individual IR analyzer are well documented. IR analysis can certainly still be used if the clinically significant difference sought between groups would be larger than the possible error (approximately 10\% of the displayed value) of the method. Any inaccuracies are less likely to have implications for studies that report $\mathrm{F}_{\mathrm{A}} / \mathrm{F}_{\mathrm{I}}$ (inspired over endexpired concentrations), because any error should affect $\mathrm{F}_{\mathrm{A}}$ and $\mathrm{F}_{\mathrm{I}}$ almost proportionally. If a particular analyzer would be over-estimating the "true" concentration by $10 \%$ for whatever reason, the ratio of $\mathrm{F}_{\mathrm{A}} / \mathrm{F}_{\mathrm{I}}$ would still be accurate: $\mathrm{F}_{\mathrm{A}} / \mathrm{F}_{\mathrm{I}}=\left(\mathrm{F}_{\mathrm{A}}^{*} 1.1\right) /\left(\mathrm{F}_{\mathrm{I}}^{*} 1.1\right)$.

\section{Conclusion}

In summary, the use of different IR absorption bands by the M-CAiOV compact multi-gas analyzer (General Electric) has allowed automated agent detection and may technically have facilitated the compensation for crosssensitivity between anesthetic vapors and other gases, but has not improved accuracy of vapor analysis beyond that of older IR analyzers. IR and GC cannot be used interchangeably, because the deviations between GC and IR mount up to $\pm 20 \%$, and because individual analyzers differ unpredictably in their performance.

\section{Competing interests}

The author(s) declare that they have no competing interests.

\section{Authors' contributions}

JH, HL, ADW, and LJS conceived the concept; HL and LJS provided the equipment and taught the analytical techniques; JH acquired the data; all authors helped prepare the manuscript, and have read and approved the final manuscript.

\section{Acknowledgements}

The M-CAiOV compact gas IR analyzers were provided by General Electric [Datex-Ohmeda], Helsinki, Finland; the calibration gases were donated by Dr. Eger El II; no financial support was provided.

The authors thank El Eger, MD, and MJ Laster, DVM, for providing the gas chromatograph and valuable comments.

\section{References}

I. Severinghaus JW, Larson CP, Eger El: Correction factors for infrared carbon dioxide pressure broadening by nitrogen, nitrous oxide and cyclopropane. Anesthesiology 1961, 22:429-32.

2. Colquhoun AD, Gray WM, Ashbury AJ: An evaluation of the Datex Normac anaesthetic agent monitor. Anaesthesia 1986, 4I: 198-204.

3. Nielsen J, Kann T, Moller JT: Evaluation of three transportable multigas anesthetic monitors: the Burel \& Kjaer Anesthetic Gas Monitor 1304, the Datex Capnomac Ultima, and the Nellcor N-2500. Clin Monitoring 1993, 9:9I-8.

4. Ishley AH, Plummer JL, Brunciman WB, Cousins MJ: An evaluation of three volatile anaesthetic agent monitors. Anaesth Intensive Care 1986, 14:437-42. 
5. Walder B, Lauber R, Zbinden AM: Accuracy and cross-sensitivity of 10 different anesthetic gas monitors. J Clin Monitoring 1993, 9:364-73.

6. Datex-Ohmeda, Helsinki, Finland, Compact Airway Modules, MIO2 June, 2005.

7. Severinghaus JW: Can large volume $\mathbf{N}_{2} \mathbf{O}$ uptake explain the second gas effect? Br J Anaesth 2006, 97:262.

8. Perma Pure LLC [http://www.permapure.com/OurTechnol ogy.htm]

9. Peyton PJ, Chong M, Stuart-Andrews C, Robinson GJ, Pierce R, Thompson BR: Measurement of anesthetics in blood using a conventional infrared clinical gas analyzer. Anesth Analg 2007, 105:680-7.

\section{Pre-publication history}

The pre-publication history for this paper can be accessed here:

http://www.biomedcentral.com/1471-2253/8/2/prepub

Publish with Bio Med Central and every scientist can read your work free of charge

"BioMed Central will be the most significant development for disseminating the results of biomedical research in our lifetime. "

Sir Paul Nurse, Cancer Research UK

Your research papers will be:

- available free of charge to the entire biomedical community

- peer reviewed and published immediately upon acceptance

- cited in PubMed and archived on PubMed Central

- yours - you keep the copyright 